\title{
Study of the Power Amplifier Transfer Function Symmetry and its Relation to Dynamic Effects
}

\author{
Zain A. Khan ${ }^{*}$, Efrain Zenteno ${ }^{*}$, Peter Händel ${ }^{\dagger}$ and Magnus Isaksson ${ }^{*}$ \\ "Dept. Electronics, Mathematics and Natural Sciences, University of Gävle, 80176 Gävle, Sweden \\ tDept. of Signal Processing, KTH Royal Institute of Technology, 10044 Stockholm, Sweden \\ zanahn@hig.se
}

\begin{abstract}
Future wireless networks will employ unprecedentedly high number of transmitters along-with larger bandwidth signals. Therefore, complexity efficient Power Amplifier (PA) modelling methods with the ability to quantify memory effects are required. This paper presents a low complexity method to quantify memory effects by studying the symmetry in the PA transfer functions estimated with the density estimation method. It is shown that the symmetry helps in reducing the method complexity by more than two orders of magnitude. Further, it is verified that the symmetry can be used as a non-model based metric for quantifying memory effects in PAs.
\end{abstract}

Keywords—power amplifier, density estimation, power amplifier modeling, symmetry, dynamics.

\section{INTRODUCTION}

The number of transmitters in modern and anticipated wireless communication systems is on the rise. Therefore, it becomes increasingly important to reduce the computational complexity incurred in modeling and compensating the amplifier output. Furthermore, the increasing bandwidth deployed in current communication standards exacerbates the memory effects appearing at the amplifier output $[1,2]$; e.g., LTE uses up to $100 \mathrm{MHz}$ [3]. In these increased bandwidth scenarios, the amount of dynamic effects at the amplifier output needs to be quantified. This will be important when designing 'optimal' (low resource and computational cost) structures for the amplifier simulation or in developing compensation techniques.

Traditional parametric models for PAs proposed in the literature $[4,5]$ depend largely on the selection of a sound model structure. Particularly, they require choosing the memory depth. Further, in widely extended polynomial based models, high nonlinear orders are coupled with memory depth. This creates a vast number of terms which significantly increases both the computational complexity [6] as well as the rate of convergence of the model. Computational complexity and rate of convergence are important parameters for real time implementations.

A method for modeling PAs is presented in [7] which estimates a nonparametric transfer function for the PA without any prior model selection. Using [7], the transfer function of the PA can be studied. In particular; we analyze and quantify its rotational symmetry [8]. The symmetry analysis of the PA transfer functions helps in significantly reducing the computational resources and storage requirements for the method in [7].

Further, although [7] considers solely the static effects of the PA output, its nonparametric nature allows to study the dynamic effects encountered at the amplifier output. We encounter a high correlation between the symmetry metric and the level of dynamic effects which shows that the symmetry metric can be reliably used to measure the amount of dynamic effects at the PA output. By removing the static part of the PA output, the level un-modeled effects caused by dynamic interactions can also be observed. Hence, by using this approach iteratively, the level of dynamic effects after including different memory depths in the structure can be quantified.

A number of techniques quantify the memory effects of power amplifiers using two tone measurements [9, 10]. However, they require specifically designed input signals to perform experiments. The proposed method works with realistic signal excitations and does not require a specific input signal. To employ real-life communication signals for low error performance, model based metrics have been proposed to quantify dynamic effects of PAs $[11,12]$. In contrast to these works, the proposed metric is nonparametric. Thus it does not rely on any model structure for the PA.

\section{BACKGROUND}

The complex valued base-band signals at the input and output of the amplifier are denoted by $u(n)$ and $y(n)$ respectively, with $n=0,1,2, \ldots, N-1$. Let the $m$ integer sample delay of $u(n)$ be denoted by $u(n-m)$. In [7], the static nonlinear transfer function of the PA is estimated by averaging over the output samples whose domain is in Euclidean vicinity of an arbitrary estimation point $x_{l}$ that spans the domain of $u(n)$. The static PA transfer function $g_{0}(\cdot)$ is estimated by [7]

$$
\hat{g}_{0}\left(x_{i}\right)=\sum_{n=0}^{N-1} \varphi\left[x_{i}, u(n)\right] y(n) \text {, }
$$


where $\varphi\left[x_{i}, u(n)\right]$ is the window averaging function which weights the samples in Euclidean vicinity of an estimation point $x_{i}$. $T$ estimation points are used to obtain the nonparametric transfer function. Since the input domain $u(n)$ is complex-valued, the $T$ estimation points span a 2 dimensional domain (real and imaginary). This increases the computational resources and storage requirements for this method. The method in [7] is similar to other statistical approaches for modelling static PAs as [13]. However, in contrast to [13], [7] uses a complex-valued input domain and generalizes the estimation process using weighted averages.

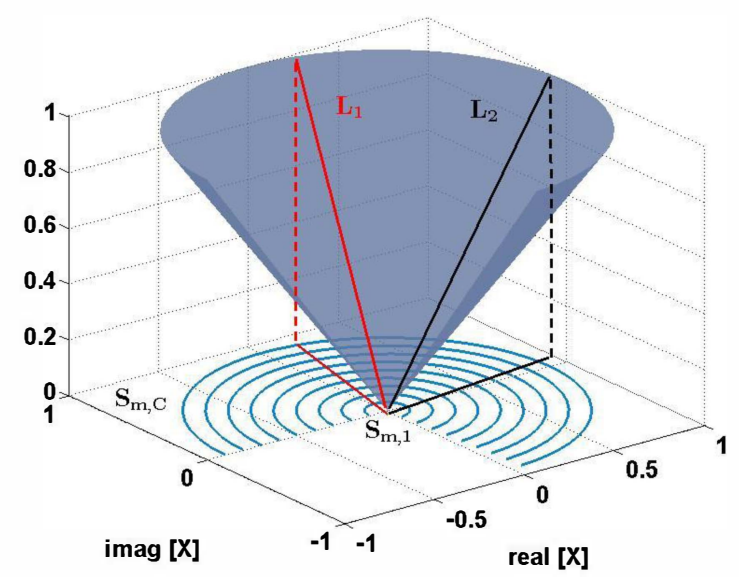

Fig. 1 Illustration of the symmetry measure $\sigma_{\mathrm{m}}$. The symmetry is the average deviation (variation) over the contours $\mathrm{S}_{\mathrm{m}, \mathrm{c}}$.

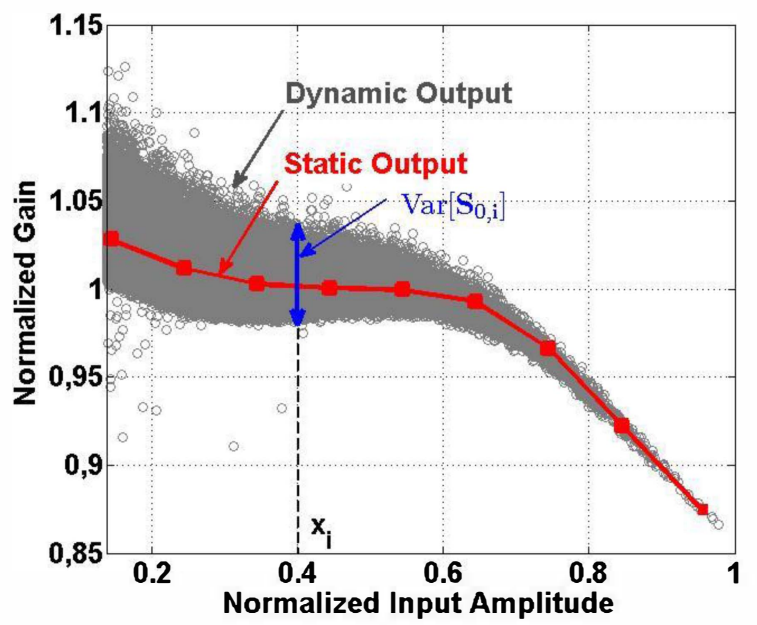

Fig. 2 Illustration of the symmetry measure as the variance to the static output caused by memory.

\section{A. Symmetry Measure}

This paper studies the rotational symmetry of $\hat{g}_{0}(\cdot)$ around the axis passing trhough the origin and perpendicular to the plane of the complex-valued input domain (cf. Fig. 1). To this end, the symmetry measure $\sigma_{0}$ is defined as the deviation of $\hat{g}_{0}(\cdot)$ compared to a perfect revolution solid obtained by rotating any radial line that belongs to $\hat{g}_{0}(\cdot)$ around the z-axis (c.f. Fig. $1)$. The symmetry measure $\sigma_{0}$ is computed as

$$
\sigma_{0}=10 \log _{10}\left\{\frac{1}{C} \sum_{c=1}^{C} \operatorname{Var}\left[S_{0, c}\right]\right\},
$$

where $S_{0, c}$ are the contours of $\hat{g}_{0}(\cdot)$ as depicted in Fig. 1. $C$ is the number of contours and $\operatorname{Var}[\cdot]$ is the variance. Thus, $\sigma_{0}$ corresponds to $-\infty \mathrm{dB}$ for high symmetry, indicating that $\hat{g}_{0}(\cdot)$ is an ideal solid of revolution. Also, any symmetry deviation is quantitatively indicated by $\sigma_{0}$.

Fig. 2 depicts a measured AM/AM curve from an amplifier; the symmetry measure can also be understood as the variance for the outputs that have the same input amplitude domain. This is illustrated in Fig. 2. Notice that the spread of measured samples in the AM/AM has been pointed as an indicator of memory effects [14]. Thus, the symmetry metric explicitly captures the spreading of the measured samples to quantify the memory effects of the PA.

\section{B. Analyzing Dynamic Effects}

By removing the static contribution from the PA output, the dynamic effects can be quantified. In particular, the transfer function of the first residual component $\hat{g}_{l}(\cdot)$, over the domain of $\mathrm{u}(n-1)$, is estimated as in (1)

$$
\hat{g}_{1}\left(x_{i}\right)=\sum_{n=0}^{N-1} \varphi\left[x_{i}, u(n-1)\right]\left[\hat{g}_{0}\left(x_{i}\right)-y(n)\right] .
$$

The nonlinear transfer function $\hat{g}_{m}(\cdot)$ takes complex valued input to produce complex valued output. In general, the transfer function $\hat{g}_{m}(\cdot)$ can be estimated over $u(n-m)$ by iteratively removing the contributions of the previous $m-1$ transfer functions. 


\section{RESULTS}

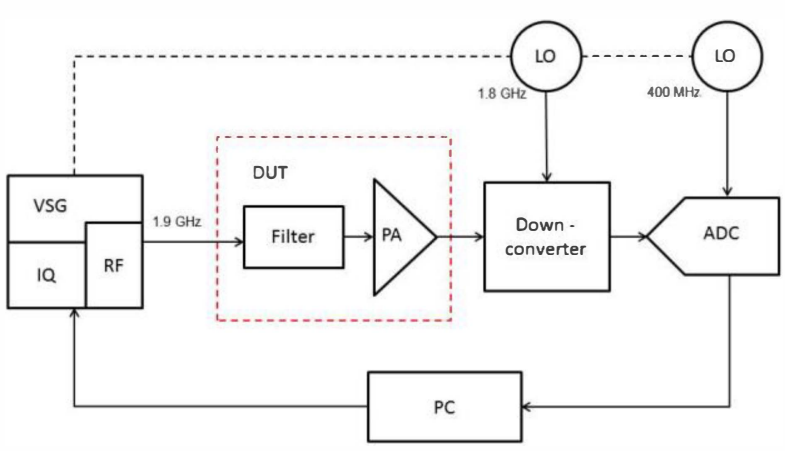

Fig.3 Block diagram of experimental setup

\section{EXPERIMENTAL SETUP}

The experimental setup is depicted in Fig.3 and comprises of a R\&S SMU 200A vector signal generator (VSG) and a wide band down-converter with a 14 bit analog-to-digital converter (ADC) having a sampling rate of $400 \mathrm{MHz}$. The $\mathrm{ADC}$ and the VSG are connected through a PC for control. The DUT is composed by the cascaded connection of a band pass filter and a PA. The filter has a pass-band of $60 \mathrm{MHz}$ centered at the operating frequency of the amplifier that is excited using the VSG.

Since the dynamic behavior of a PA depends upon the signal bandwidth at its input $[1,2]$, the deployed band-pass filter exacerbates the dynamic effects encountered in the DUT. By generating excitation signals of different bandwidths, we study the relationship between deviations in symmetry of the PA transfer functions and the observed DUT dynamic effects.

TABLE I. CHARACTERISTICS OF THE PAS UNDER CONSIDERATION

\begin{tabular}{|c|c|c|c|}
\hline Manufacturer & Type & $\begin{array}{c}\text { Gain } \\
{[\mathbf{d B}]}\end{array}$ & $\begin{array}{c}\mathbf{P}_{\mathbf{1 d B}} \\
{[\mathbf{d B m}]}\end{array}$ \\
\hline 1 & Unknown & 52 & 35 \\
\hline 2 & Doherty & 14 & 46 \\
\hline 3 & $\mathrm{AB}$ & 16 & 48 \\
\hline
\end{tabular}

TABLE II. SYMMETRY MEASURE OF THE PA - STATIC TRANSFER FUNCTION AND THE NMSE ACHIEVED WITH THE GENERAL AND AMPLITUDE DEPENDENT APPROACHES FOR DIFFERENT PAS

\begin{tabular}{|c|c|c|c|}
\hline PA & $\begin{array}{c}\text { Symmetry } \\
\text { Measure } \\
\boldsymbol{\sigma}_{0}[\mathbf{d B}]\end{array}$ & $\begin{array}{c}\text { NMSE }[\mathbf{d B}] \\
\text { General } \\
(\mathbf{T}=\mathbf{4 9 0 0})\end{array}$ & $\begin{array}{c}\text { NMSE }[\mathbf{d B}] \\
\text { Amplitude } \\
\text { Dependent }(\mathbf{T}=\mathbf{3 5})\end{array}$ \\
\hline Unknown & -52.8 & -30.1 & -30.4 \\
\hline Doherty & -51.7 & -34.4 & -34.6 \\
\hline $\mathrm{AB}$ & -59.9 & -50.1 & -50.0 \\
\hline
\end{tabular}

\section{A. Complexity Reduction}

The symmetry measure $\sigma_{0}$ of the static transfer function $\hat{g}_{0}(\cdot)$ is computed for three different PAs excited by an OFDM (Orthogonal Frequency Division Multiplexing) signal of 4 $\mathrm{MHz}$ bandwidth and an approximate PAPR of $11 \mathrm{~dB}$. The commercial PAs considered in this paper are listed in Table I. Since $\sigma_{0}$ is an average over the number of contours, $C$ should be sufficiently large for numerical purposes. Hence, for the results in this paper, we use $C=300$ in (2). Further, to compute the symmetry measure we use a very narrow window averaging function in (1) to avoid a bias in the estimation of $\hat{g}_{0}(\cdot)[15]$.

Table II shows the symmetry measure $\sigma_{0}$ for all the PAs. The level of $\sigma_{0}$ is below $-50 \mathrm{~dB}$ (in the order of $10^{-5}$ ) which suggests that the PAs under consideration observe rotational symmetry. Hence, from (1), it can be concluded that $\hat{g}_{0}(\cdot)$ can be described by a perfect solid of revolution around the $\mathrm{z}$-axis and consequently it is possible to estimate $\hat{g}_{0}(\cdot)$ over a diagonal of the input domain only. This helps in reducing the number of entries in the nonparametric estimation method [7] by a factor of $2 \sqrt{T}$. For instance, the number of entries will reduce from the full method to its amplitude approach from 4900 entries to 35. This is a reduction of more than two orders of magnitude. This, in turn, not only simplifies the estimation process but the manipulation and storage of the data as well.

Normalized Mean Squared Error (NMSE) for the three PAs is also computed with respect to both the general (amplitude and phase dependent as in [7]) and amplitude dependent transfer functions in Table. II. It can be observed that there is no loss in NMSE between the two model approaches for all measured PAs. This result confirms the modeling of PAs by complexity efficient amplitude dependent transfer functions [5].

\section{B. Dynamics}

Fig. 4 depicts the symmetry measure $\sigma_{0}$ of the static transfer function $\hat{g}_{0}(\cdot)$ against its NMSE performance for the class $\mathrm{AB}$ PA excited with noise-like signals of different bandwidths. A linear relationship can be observed as indicated in the dashed line for comparison. Since the PA transfer function $\hat{g}_{0}(\cdot)$ describes the static contribution, degradations in NMSE are attributed to dynamic effects. It can be observed in Fig. 3 that the larger the signal bandwidth the greater the dynamic effects as depicted by the degradation in NMSE. Deviations in symmetry follow a similar trend with respect to increasing bandwidths where larger dynamic effects are expected due to the filter placed prior to the PA. The correlation between the NMSE and the symmetry measure $\sigma_{0}$ is above $99 \%$ which shows that the symmetry measure can be reliably used to quantify dynamic effects in the PA.

Fig. 5 depicts the symmetry measure $\sigma_{0}$ of the PA transfer function $\hat{g}_{0}(\cdot)$ against its NMSE performance for all the PAs listed in Table I excited with noise-like signals of different bandwidths. Similar to the results in Fig. 3, it is observed that all PAs follow the linear trend between degradations in NMSE 
and the symmetry measure $\sigma_{0}$ with increasing bandwidth for the static transfer function transfer function $\hat{g}_{0}(\cdot)$. The linear relationship between the NMSE and $\sigma_{0}$ is not identical for all PAs. However, the linear trend for all PAs has the same slope. This result shows that the amount of dynamics introduced with increasing signal bandwidth is the same for all PAs due to the pass band of the filter. This further validates the use of the symmetry measure as a metric to quantify dynamic effects.

Fig. 6 depicts the amplitude of $\hat{g}_{0}(\cdot)$ and $\hat{g}_{l}(\cdot)$ for the class $\mathrm{AB}$ PA with $80 \mathrm{MHz}, 20 \mathrm{MHz}$ and $4 \mathrm{MHz}$ signals respectively. The static transfer function $\hat{g}_{0}(\cdot)$ for the $80 \mathrm{MHz}$ signal has the lowest symmetry and hence the largest contributions of dynamic effects while the transfer functions for the $4 \mathrm{MHz}$ signal observe the highest symmetry and are dominated by static effects as a result. Furthermore, the remaining dynamic effects can be observed by the symmetry measure $\sigma_{1}$ of the residual transfer functions $\hat{g}_{l}(\cdot)$.

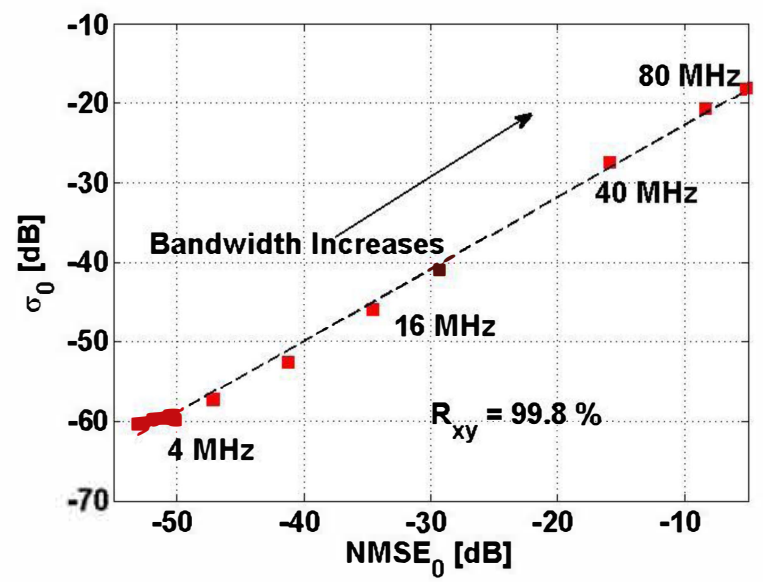

Fig 4. Symmetry measure $\sigma_{0}$ versus the NMSE considering only the static contribution in the class $\mathrm{AB}$ PA output for different bandwidths of the excitation signal.

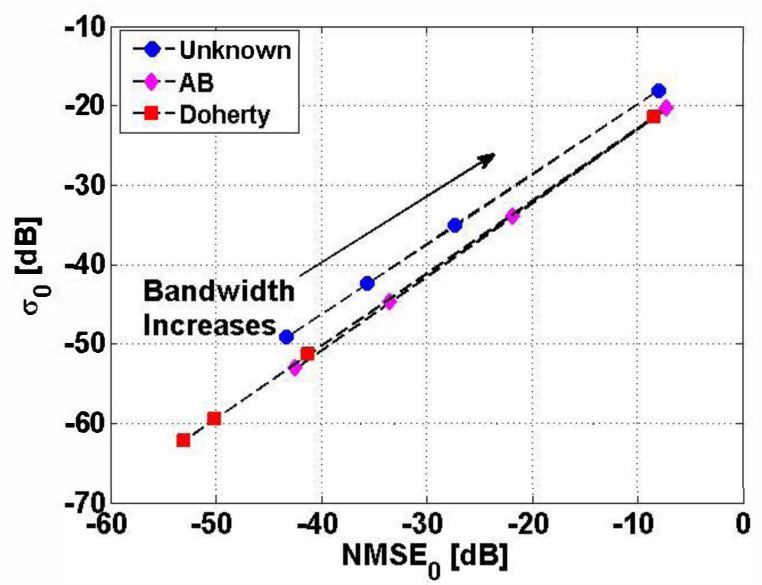

Fig 5. Symmetry measure $\sigma_{0}$ versus the NMSE considering only the static contribution for all the PAs under consideration for different bandwidths of the excitation signal.
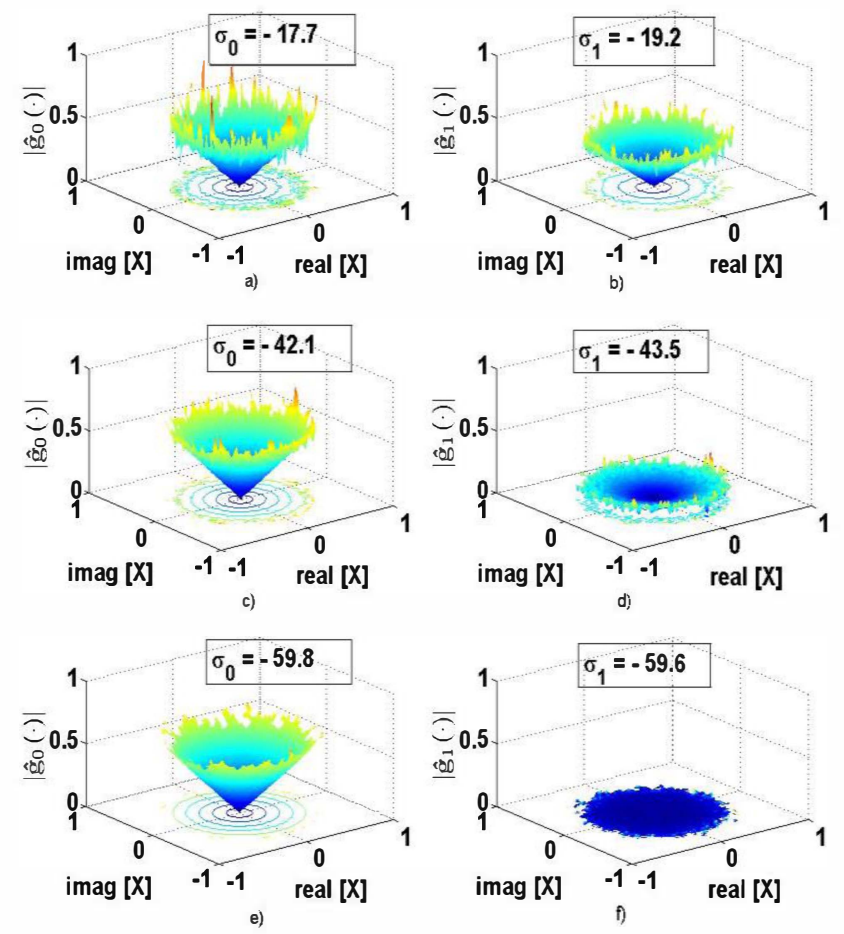

Fig 6. Amplitude of $\hat{g}_{0}(\cdot)$ and $\hat{g}_{l}(\cdot)$ for the class AB PA obtained from different bandwidths of the excitation signal. $80 \mathrm{MHz}$ (a and b), $20 \mathrm{MHz}$ (c and d) and $4 \mathrm{MHz}$ (e and $\mathrm{f}$ ).

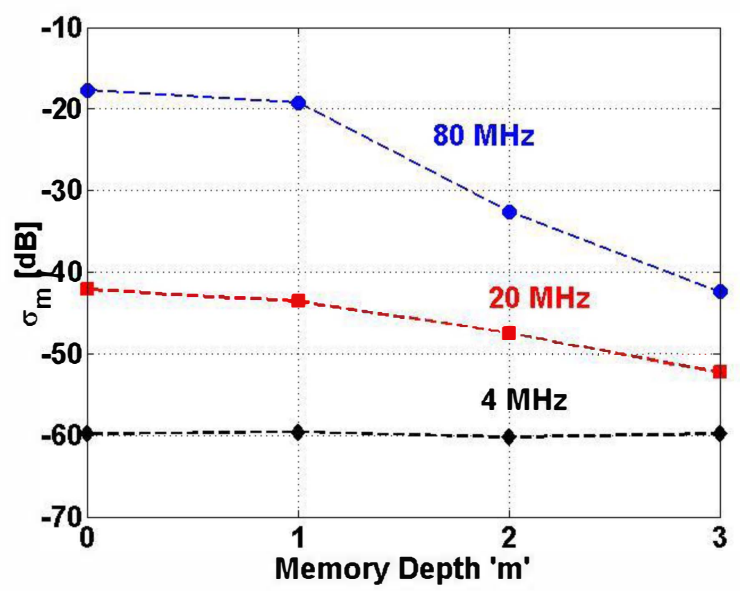

Fig. 7. Symmetry measure $\sigma_{\mathrm{m}}$ versus the memory depth for the class AB PA with different bandwidth signals. 
Fig. 7 plots the symmetry measure $\sigma_{\mathrm{m}}$ against the memory depth for the class AB PA with different bandwidth signals. The significance of the dynamic effects is indicated by the level of $\sigma_{\mathrm{m}}$. It can be observed that $\sigma_{\mathrm{m}}$ does not change for the $4 \mathrm{MHz}$ signal since it is dominated by static effects. For the 20 and $80 \mathrm{MHz}$ signals, there is a decrease in the symmetry measure $\sigma_{\mathrm{m}}$ with increasing memory depth which is proportional to the residual dynamic effects. Hence, the level unmodelled dynamic effects can be discovered using the symmetry measure $\sigma_{\mathrm{m}}$.

\section{DISCUSSION}

The density estimation method is average-based; it requires a sufficient number of measurements samples to provide reliable estimates. Estimation with few samples gives lower accuracy, which may happen for signals with tailed distributions, where higher amplitudes are unlikely to occur. On the other hand, real time running averages present low storage and computational power but can include large amount of measurement samples in the identification process. Hence, this method can be attractive for real time implementations.

It can be noted that density estimation helps in analyzing the symmetry of the PA transfer functions and its relationship to signal bandwidth. However, further research studies are required to understand other factors affecting this property.

\section{CONCLUSION}

A low complexity nonparametric metric for quantifying memory effects has been proposed and verified in this paper. The complexity of the density estimation method is significantly reduced by analyzing the symmetry of the PA transfer functions while maintaining its error performance. We show that the dynamic effects created by the bandwidth increase are highly correlated with the proposed metric. This confirms the use of symmetry as a tool for quantifying dynamic effects in PAs.

\section{REFERENCES}

[1] M. Isaksson, D. Wisell and D. Rönnow, "A comparative analysis of behavioral models for RF power amplifiers," IEEE Trans. Microw. Theory Tech., vol. 54, no. 1, pp. 348-359, Jan. 2006.

[2] T.Cunha, E.Lima and J.Pedro, "Validation and physical interpretation of the power amplifier polar Volterra model," IEEE Trans. Microw. Theory Tech., vol. 58, no. 12 , pp. 4012-4021, Dec. 2010.

[3] T. Ali-Yahiya, Understanding LTE and its Performance, New York, USA: Springer, 2011

[4] H.Ku and J.S.Kenney, "Behavioral modeling of nonlinear RF power amplifiers considering memory effects," IEEE Trans. Microw. Theory Tech., vol. 51, no. 12 , pp. 2495-2504, Dec. 2003.

[5] D.Morgan, Z.Ma, J. Kim, M. Zierdt and J.Pastalan, "A generalized memory polynomial model for digital predistortion of RF Power Amplifiers," IEEE Trans. Signal Process., vol. 54, no. 10 , pp. $3852-$ 3860, Oct. 2006.

[6] A. Tehrani,H. Cao, S. Afsardoost, T. Eriksson, M. Isaksson and C. Fager, "A copaprative analysis of the complexity/accuracy tradeoff in power amplifier behavioral models," IEEE Trans. Microw. Theory Tech., vol. 58, no. 6, pp. 1510-1520, Jun. 2010.

[7] Z.A.Khan, E. Zenteno, M. Isaksson and P. Händel, "Density estimation models for strong nonlinearities in RF power amplifiers,"in APMC Asia Pacific Microw. Conf., Sendai, pp-, Nov. 2014.

[8] C. Dryzun and D.Anvir, "Generalization of the continuous symmetry measure: the symmetry of vectors, matrices, operators and functions," Phys. Chem. Chem. Phys., vol. 11, no. 42, pp. 9653-9666, Aug. 2009.

[9] Hyunchul Ku; McKinley,M.D.; Kenney J.S., "Quantifying memory effects in RF power amplifiers," IEEE Trans. Microw. Theory Tech., vol. 50, no. 12 , pp. 2843-2849, Dec. 2002.

[10] Vuolevi, J.H.K.; Rahkonen, T.; Manninen, J.P.A., "Measurement technique for characterizing memory effects in RF power amplifiers," IEEE Trans. Microw. Theory Tech, vol.49, no.8, pp.1383-1389, Aug 2001

[11] P.Landin, M. Isaksson and P.Händel, "Comparison of evaluation criteria for power amplifier behavioral modeling," in IEEE MTT-S Int. Microw. Symp. Dig., pp. 1441-1444, Jun. 2008.

[12] W.Bosch and G.Gatti, "Measurement and simulation of memory effects in predistortion linearizers," IEEE Trans. Microw. Theory Tech., vol. 37, no. 12 , pp. 1885-1890, Dec. 1989.

[13] D. Huang, X. Huang, and H. Leung, "Nonlinear compensation of highpower amplifier distortion for communication using a histogrambased method," IEEE Trans. Signal Process, vol. 54, no. 11, pp. 4343-4351, Nov. 2006.

[14] Taijun Liu; Boumaiza, S.; Ghannouchi, F.M., "Deembedding static nonlinearities and accurately identifying and modeling memory effects in wide-band RF transmitters," IEEE Trans. Microw. Theory Tech., vol.53, no.11, pp.3578-3587, Nov. 2005.

[15] E. W. Bai, "Non-Parametric Nonlinear System Identification: A Data-Driven Orthogonal Basis Function Approach," IEEE Autom. Control., vol. 53, no. 11, pp. 2615-2626, Dec. 2008. 
